\title{
Fabrication of Multiple Layered Scaffolds with Controlled Porous Micro-Architecture
}

\author{
Chunrong Yang ${ }^{a, b *}$, Huazhong Wu ${ }^{c}$, Jianhua Wang ${ }^{d}$ \\ ${ }^{a}$ School of Materials Science and Engineering, Fujian University of Technology, Fuzhou, Fujian \\ Province, China \\ ${ }^{b}$ Fujian Provincial Key Laboratory of Advanced Materials Processing and Application, Fuzhou, Fujian \\ Province, China \\ ${ }^{c}$ Department of Chemistry and Chemical Engineering, University of Minjiang, Fuzhou, Fujian \\ Province, China \\ ${ }^{d}$ Institute of Biomedical and Pharmaceutical Technology, Fuzhou University, Fuzhou, Fujian Province, \\ China
}

Received: September 21, 2016; Revised: November 22, 2016; Accepted: December 19, 2016

\begin{abstract}
A multi-layer scaffold that incorporates bioglass, phosphatidylserine and steroidal saponins loaded collagen microparticles was prepared in this study. Combinatorial processing techniques involving porogen leaching protocol and freeze-drying were used. Drug distribution and microstructure could be controlled mainly by the concentrations of drug-loaded collagen microparticles and porogen as well as freezing temperature for different layers of the scaffolds. Morphological observation, measurement of swelling properties, examination of drug release kinetics, and analysis of cell bioactivity showed that the resultant scaffolds had highly interconnected pores, gradient drug distribution and graded porous micro-architecture, similar swelling ratio for different layers, better drug release kinetics in view of lower initial release and slower average release rate compared to the scaffolds with homogeneous structure, as well as approving support of cell ingrowth. This study suggests that the optimized scaffolds have promising potential for applications in bone tissue engineering.
\end{abstract}

Keywords: multi-layer scaffold, microstructure, drug distribution

\section{Introduction}

To date, most approaches related to tissue engineering involve delivery of drugs via porous scaffolds for regeneration of tissues, and thus, the architecture of scaffolds has a decisive effect on the load and release of drugs. Complex and controlled architectures are often a necessary alternative to loading drugs in a porous scaffold, as control over structure is critical for gaining adequate release function. Recent novel techniques have been reported for achieving these complex architectures, including electrospinning, phase separation, and precipitation as well as gas foaming/particulate leaching ${ }^{1-4}$. Besides that, several groups have also investigated the layering of 2-D sheets to build up a complex 3-D architectures with porous features $^{5-8}$. Most of these techniques result in scaffold with order structure.

However, it has been increasingly recognized that these delivery systems should deliver drugs in a spatiotemporal way, including that (1) burst release should be lessened and (2) stable release should sustain a relatively long time to obtain the desired effects. It is therefore critical to design the scaffold to provide inhomogeneous structure for safe and stable drug release.

Based on the compositions of nature bone, naturally derived collagen (COL) has the potential advantages

* e-mail: lambmeo@163.com of specific cell interactions and hydrophilicity. Phosphatidylserine (PS) has been implicated in variety of cellular processes such as cell migration, cell adhesion, tumor metastasis, cell morphogenesis and differentiation, and wound healing. Selected compositions of silicate and phosphate glasses have been proved to react with physiological fluid and form tenacious bonds to hard and soft tissues through cellular activity. Therefore, bioglass (BG), COL and PS, when processed into biomaterial, will result a bone scaffold with excellent mechanical properties, appropriate bio-degradation, and well biocompatibility and bioactivity, which has been demonstrated in our previous work 9 .

In this report, we applied combinatorial processing techniques involving porogen leaching protocol and freeze-drying to develop a novel, multi-layered scaffold using BG, PS and steroidal saponins (SS)-loaded collagen microparticles. As the major pharmacological constituents of ophiopogon japonicus, a traditional Chinese medical herb, SS are osteoinductive and can be used to treat bone and joint disease $\mathrm{s}^{10}$. The resulting scaffolds were designed to have gradient pore structure and drug distribution. Additionally, the construct was evaluated with respect to microstructure, swelling parameters, drug release kinetics, and cell bioactivity to demonstrate the potential utility for bone tissue engineering. 


\section{Materials and methods}

\subsection{Materials}

58s-BG were synthesized through sol-gel method and consisted of $60 \mathrm{wt} \% \mathrm{SiO}_{2}, 35 \mathrm{wt} \% \mathrm{CaO}$ and $5 \mathrm{wt} \% \mathrm{P}_{2} \mathrm{O}_{5}$. The steroidal saponins-loaded collagen microspheres were prepared using a water-in-oilin-water emulsion/solvent evaporation technique ${ }^{11}$. PS and NuSieve GTG agarose gel were supplied by Boao Medical Ltd. (Shanghai, China) and Liyuan Medical Ltd. (Xiamen, China), respectively. Lyophilized steroidal saponins were obtained from the College of Pharmacology, Shandong University of Traditional Chinese Medicine (Jinan, China). Dulbecco's minimum essential medium (DMEM) and fetal bovine serum (FBS) were purchased from Sigma (St. Louis, MO, USA). 1-ethyl-3-(3-dimethyl aminopropyl) carbodiimide (EDC), N-hydroxysuccinimide (NHS), morpholine ethanesulfonic acid (MES) and other reagents were of analytical grade.

\subsection{Construction of multiple layered BG-COL-PS scaffolds}

BG-COL-PS scaffolds were prepared as follows. In brief, paraffin spheres of 180-250 $\mu$ m diameter and steroidal saponins-loaded collagen microspheres of around $100 \mu \mathrm{m}$ diameter were filled into cylindrical syringe prior to heating the spheres at $37^{\circ} \mathrm{C}$. After annealing to room temperature, nano-bioglass powder and phosphatidylserine solution were respectively added to the syringe and carefully aspirated to fill the interspace between the spheres. The mixture were then shock-frozen as a thin layer (around $2 \mathrm{~mm}$ in thickness). After the layer was nearly solidified, laminated assembly was repeated and a new layer containing a same amount of BG nanoparticles, lower weight ratio of PS, higher weight ratio of drug-loaded collagen and paraffin microspheres were spread onto the top of the solidified layer. Then, the third layer was prepared with same composition ratio to the first layer. The triple layered scaffolds were attained via such additional layer-supraposition. Then the scaffolds were freeze dried at $-30^{\circ} \mathrm{C}$ for $45 \mathrm{~h}$.

After being lyophilized, the scaffolds were treated with $90 \%(\mathrm{v} / \mathrm{v})$ methanol solution for $45 \mathrm{~min}$ and then treated with hexane for $20 \mathrm{~h}$ to extract the paraffin spheres. Hexane was replaced after $24 \mathrm{~h}$ and the resulting scaffolds were dried under vacuum for $10 \mathrm{~h}$ in order to completely dry the scaffolds and minimize residual hexane. Then, cross-linking was performed in MES buffer ( $\mathrm{pH}$ 5.5) with EDC. NHS was added in an EDC:NHS ratio of 4:1. After crosslinking with a reaction time of $4 \mathrm{~h}$ the samples were washed twice with $0.1 \mathrm{M} \mathrm{Na}_{2} \mathrm{HPO}_{4}$ for $1 \mathrm{~h}$ and 4 times with deionized water for $30 \mathrm{~min}$. Finally, the scaffolds were dried in vacuum.

For the purpose of comparison, another type of scaffolds (Type 2) with homogeneous microstructure and durg distribution were also prepared following the same procedure. The total amounts of compositions and paraffin microspheres were same to the inhomogeneous scaffolds (Type 1), which consisted of $65 \%$ parts of inorganic component BG by weight and $35 \%$ part of organic components, including $80 \mathrm{wt} . \%$ drug-loaded collagen microspheres, and $20 \mathrm{wt} . \%$ PS. The weight ratio of paraffin microspheres to scaffolds was 1:3.

\subsection{Characterization}

\subsubsection{Morphology observation}

To observe the morphology of each layer of the scaffolds, disk samples ( $10 \mathrm{~mm}$ in diameter) were cut into approximately isopachous sheets with $2 \mathrm{~mm}$ of thickness. Scanning electron microscopy (SEM) (S-3400N, Hitachi Co., Tokyo, Japan) was used and the sheets were sputter-coated with gold for observation at $10 \mathrm{kV}$ and varying levels of magnification.

\subsubsection{Porosity testing}

To determine porosity of each layer, disk sheets with 10 $\mathrm{mm}$ in diameter and $2 \mathrm{~mm}$ in thickness were tested using amercury intrusion porosimeter (AutoPore IV).

\subsubsection{Swelling kinetics measurements}

Swelling kinetics measurements were based on the determination of the volume of retained low-molecular penetrating agent $(0.5 \mathrm{M}$ solution of acetic acid $)$ dependent on the time of swelling at a temperature $25^{\circ} \mathrm{C}$. Disk samples were used, and each weighed $1.0 \mathrm{~g}$.

\subsubsection{Examination of drug release kinetics}

The drug release experiment of the two types of scaffolds was performed in a continuous flow system which was made in our own laboratory. Phosphate buffered saline (PBS) was employed for the release medium.

After specific time periods, the specimens were removed from the PBS fluids and the supernatant was collected. The drug content of supernatant for each interval was determined by using the ultra performance liquid chromatography (UPLC, Waters Co., Milford, USA).

\subsubsection{Analysis of cell bioactivity}

To study the effect of pore size and porosity of inhomogeneous scaffolds on osteoblast biocompatibility, MC3T3-E1 cells (Japan Riken Cell Collection) were chosen and seeded onto whole (10 $\mathrm{mm}$ in diameter and $6 \mathrm{~mm}$ in thickness) or each layer (10 $\mathrm{mm}$ in diameter and $2 \mathrm{~mm}$ in thickness) of inhomogeneous scaffolds respectively at a concentration of 15,000 cells $/ \mathrm{cm}^{2}$ in DMEM-10\%-FBS medium. 
Prior to cell culture assays, all samples were soaked in PBS overnight under UV to wet and sterilize them followed by a 20 min soak in media to allow for the deposition of serum proteins.

The proliferation kinetics of MC3T3-E1 cells on different layers of the scaffolds was assessed using a 3-(4,5-dimethyl2thiazolyl)-2,5-diphenyl-2H tetrazolium bromide (MTT) (Sigma Co., Saint Louis, USA) assay[9]. Briefly, the cells and samples were remain undisturbed in a humidified incubator $\left(37^{\circ} \mathrm{C}\right.$ and $\left.5 \% \mathrm{CO}_{2}\right)$ for $1,3,7,14$ and 21 days. After incubation, the sheets were rinsed twice with PBS and $200 \mu \mathrm{L}$ MTT, $1.8 \mathrm{ml}$ medium was added to each sheet for incubation at $37{ }^{\circ} \mathrm{C}$ for $4 \mathrm{~h}$. Then the MTT solution was removed and insoluble blue formazan crystal was dissolved in dimethyl sulfoxide (DMSO). One hundred microlitres of solution from each sheet was aspirated and poured in a 96well plate for absorbance measurement using a microplate spectrophotometer at a wavelength of $540 \mathrm{~nm}$. Non-seeded sheets were applied as negative control. The MTT absorbance data were converted into cell numbers based on the standard curve obtained at the same time.

The behaviors of cell attachment and growth were observed using a phase-contrast microscope (Olympus IX 71, Tokyo, Japan).

\subsubsection{Statistical analysis}

The data were reported as the mean $\pm \mathrm{SD}$. Two-factor analyses of variance (ANOVAs) with Bonferroni-Dunn and Fisher's post hoc tests for multiple comparisons as well as Student's $t$ test were performed using Sigma StatTM Software for Windows Version 2.03, SPSS Inc. Significance was accepted at a level of $\mathrm{P}<0.05$.

\section{Result and discussion}

\subsection{Fabrication of multi-layer scaffolds}

To achieve multiple layered scaffolds with controlled porous micro-architecture while lessening possibly burst release of drugs, combinatorial processing techniques involving freeze-drying and adjustable gradients of paraffin spheres and compositions were developed. In the process of fabrication, pores inside scaffolds were usually generated by cryogenically removing ice crystallite formed at a fixed freezing temperature, and porosity and average pore size were mainly controlled by concentration of paraffin spheres, freezing temperature and freezing rate.

By changing concentration of paraffin spheres, vertical pore gradients from the top layer to the bottom layer inside scaffolds were achieved, and this would potentially create multi-scale freezing cubicles for multiple layers. Therefore, combining variable concentrations of paraffin spheres with freeze-processing method, micro-architecture of each layer would be effectively limited and controlled.
It is known that bone implantation should can resist against enzymatic degradation with enough strength during bone regeneration. In terms of this scaffolds, the disadvantages of using high content of collagen are low biomechanical stiffness and rapid biodegradation. The high enzymatic turnover rate of natural collagen in vivo makes stabilization of collagen-based biomaterials necessary. This could be achieved by chemical crosslinking methods, which could provide this scaffolds with desired mechanical properties for implantation and defect repair. In this study, the scaffolds have been crosslinked with EDC/NHS. Use of EDC and NHS could yield this scaffolds with good biocompatibility, higher cellular differentiation potential and with increased resistance against enzymatic degradation.

\subsection{Layered scaffolds with gradient pore structures}

In this study, a type of triple layered and drug-loaded BG-COL-PS scaffolds was constructed. Scanning electron microscopy observation of the triple layers of network composite scaffolds showed a continuous structure of irregular interconnected pores (Figure 1). The pore sizes ranged from several microns up to about $400 \mu \mathrm{m}$. In addition, there were no any obvious clefts were observed in the interface zone between adjacent layers.

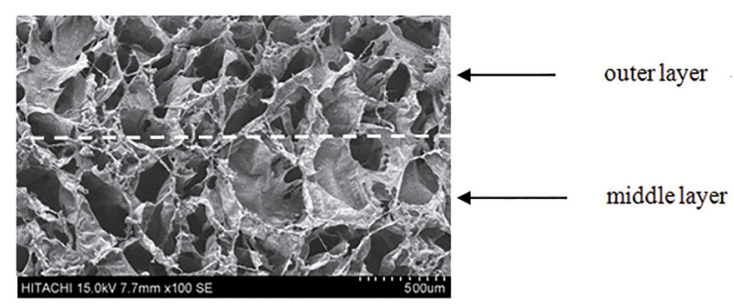

Figure 1: SEM image for interface inside the porous multi-layer scaffolds. Dotted white line indicates interface zone.

The top layer and bottom layer were similar in compositions and pore structure, but the middle layer was endowed with greater average pore size, higher porosity and drug content. The inner porous structure of the scaffolds can be controlled by varying the solid content of the paraffin spheres and freeze-drying conditions (Table 1). As the ratio of paraffin spheres and the freezing temperature lowered, the density of freeze-dried scaffolds increased, and the porosity and average pore size lowered.

Besides the influence of paraffin content, the differences in the porosity and average pore size also reflect the differences in heat transfer rates during the freezing process of the scaffolds. It is possible that at a higher freeze temperature, the number of nuclei of ice crystallization initially formed is smaller than that at a lower freezing temperature, leading to an increased final size of ice crystals. Since the larger ice 
Table 1: Characteristic of composite scaffolds

\begin{tabular}{lcccc}
\hline Paraffin ratio & Quenching temperature & Density & Average pore size & Porosity \\
\hline wt $\%$ & ${ }^{\circ} \mathrm{C}$ & $\mathrm{kg} \cdot \mathrm{m}^{-3}$ & $\mu \mathrm{m}$ & $\mathrm{v} \%$ \\
30.0 (Type 1, top layer) & -60 & $79.84 \pm 0.21$ & $175 \pm 30$ & $86.1 \pm 0.8$ \\
40.0 (Type 1, middle layer) & -60 & $78.25 \pm 0.14$ & $185 \pm 40$ & $88.5 \pm 0.4$ \\
30.0 (Type 1, bottom layer) & -60 & $80.12 \pm 0.19$ & $170 \pm 35$ & $85.8 \pm 0.6$ \\
40.0 (Type 1, middle layer) & -40 & $76.20 \pm 0.24$ & $280 \pm 60$ & $89.3 \pm 0.4$ \\
40.0 (Type 1, middle layer) & -70 & $79.18 \pm 0.22$ & $100 \pm 35$ & $86.7 \pm 0.5$ \\
33.3 (Type 2) & -60 & $78.12 \pm 0.23$ & $180 \pm 25$ & $87.5 \pm 0.3$ \\
\hline
\end{tabular}

crystals push to expand biopolymer chains to a greater extent, the pore size of scaffolds will be increased. On the contrary, it is possible that a rapid cooling causes the formation of many ice crystals nuclei, resulting in the formation of smaller-sized pores.

\subsection{Swelling ratio}

The homogeneous scaffolds and different layers of inhomogeneous scaffolds were compared morphologically with respect to their formal stability using swelling ratios and their volume changes after being soaked with $0.5 \mathrm{M}$ solution of acetic acid. Swelling is the process in which collagen and phosphatidylserine receives liquid and simultaneously enlarges its volume. Swelling of the composite scaffolds was generally observed in two steps. Acetic acid attacked first of all (it was saturation of the structure), and the second step can be explained by the structure opening after break of physical bonds, particularly in a free collagen structure, but not in conjugate between collagen and phosphatidylserine due to crosslinking. We can state that the conjugate remains stable in the acidic environment, which was especially aggressive for collagen. The conjugate provided anchoring of biogalss particles in the structure of the composite, and cohesion of the material.

The behavior of scaffolds during swelling can be the result of simultaneous influenced by at least two antagonistic factors: the polarity of the composite material and the formation of hydrogen bonds between the macromolecular components. The polarity of the bioglass material influences swelling positively. In the other case, hydrogen bonds influence the swelling negatively, which is the result of the creation of ion complexes between the carboxylate groups of phosphatidylserine and amino groups of collagen.

It can be seen from Figure 2 that there were not significant differences in swelling ratio for all sheets, which may be ascribed to two contradictory factors: on the one hand, higher porosity and greater pore size shoud mean more liquid for the matching layer, which leaded to greater swelling; on the other hand, both collagen and phosphatidylserine are hydrophilic biomolecules because of their polar groups. Therefore, even though a certain amount of crosslinker was

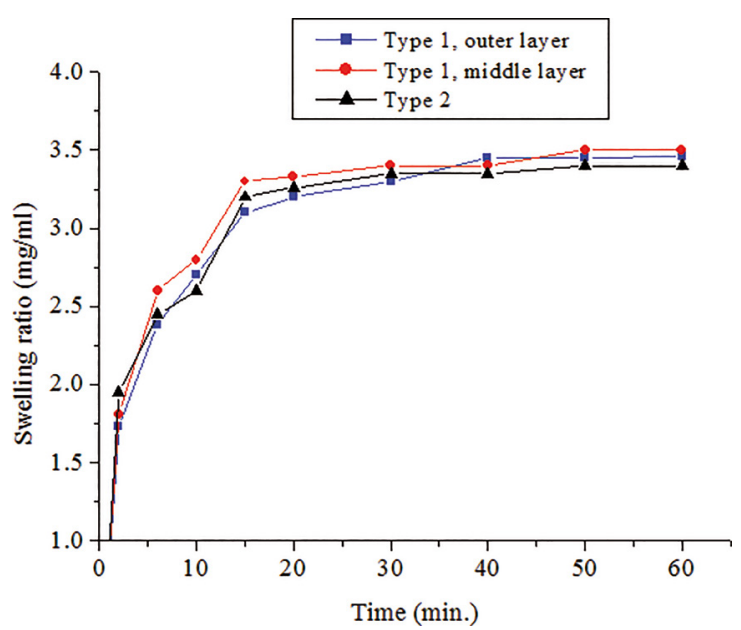

Figure 2: Swelling ratio of two types of scaffolds with different microstructure and drug distribution

used, the two types scaffolds still swelled to some extent. However, the contents of organic components, including collagen and phosphatidylserine were set to decrease with the increase of paraffin spheres which caused higher porosity and greater pore size inside the scaffolds. These results indicate that presently developed inhomogeneous or homogeneous scaffolds have similar swelling characteristics.

\subsection{Drug release from the multiple layered scaffolds}

The effect of the structure of the scaffolds on the release kinetics of drugs were evaluated as shown in Figure 3. The release profile in the homogeneous scafflods was triphasic consisting of (1) a typical burst release within the first $12 \mathrm{~h}$, followed by (2) a period of low release rates until day 7 , and finally (3) a marked increase in release from these days on.

In the case of the scaffolds with gradient structure, it was noticed that initial release within the first $12 \mathrm{~h}$ was measurably lower with $22.34 \pm 0.72 \%$ when respectively comparing to the homogeneous scaffolds with $31.32 \pm 1.21 \%$. Subsequently, the drugs were released in a continuous and slow pattern. Besides that, the average release rate of drugs 


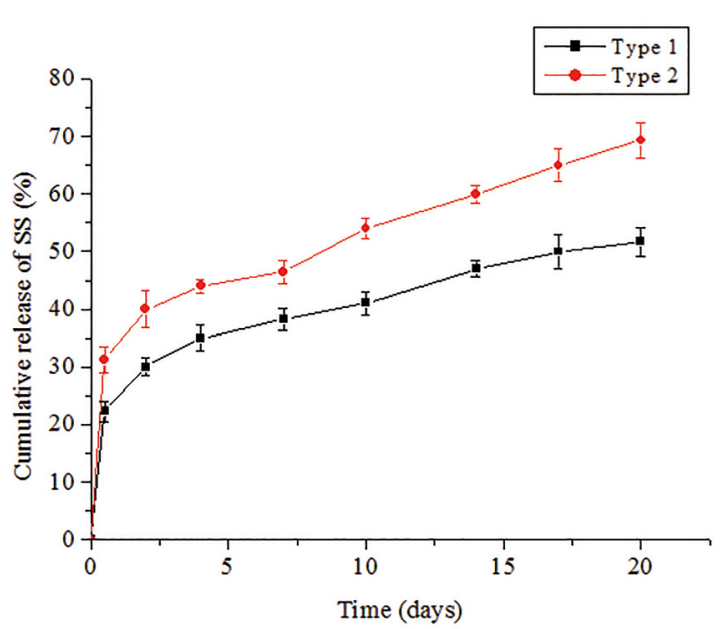

Figure 3: Cumulative release of SS from two types of scaffolds with diffrent microstructure and drug distribution

from the homogeneous scaffolds was faster significantly than that of the inhomogeneous scaffolds. The cumulative release of drug from the inhomogeneous scaffolds was $51.75 \pm 0.41 \% \%$ in 20 days, while drug released from the homogeneous scaffolds reached $69.34 \pm 0.25 \% \%$.

The different release profiles of drugs from the two types of scaffolds have been largely affected by the structure and drug distribution. This outcome was explained to result from the diffusional barrier through the scaffold matrix. In the case of the scaffolds with gradient structure, when PBS fluid flowed from the top layer to bottom layer, drug diffusion was driven from low to high resistance, which induced slow release. In contrast, diffusion from the homogeneous structure was faster. Additionally, the encapsulated drugs were absorbed on the middle layer much more than the outer two layers. The form would enhance the therapy efficiency and vary release profile in comparison with traditional incorporation methods.

Such differences in release kinetics may have distinct effects on bone regeneration. A key goal of sustained delivery techniques is achieving therapeutic levels of released factors over biologically relevant time scales. Longer-lasting and lower release amount within the safe range could avoid potential toxicity and reduce undesirable side-effects, such as metaplasia, hyperplasia, hypertrophy, unwanted vessel growth, or ectopic bone formation. This leads to the conclusion that the constructed inhomogeneous scaffolds incorporating gradient drug-loaded microparticles could allow spatially effective release of drug, a prerequisite for the regeneration of bone defects.

Additionally, the release dose and period could be controlled by changing the pore structure and content of drug-loaded collagen microparticles for different layers. Therefore, such a sustained delivery vehicle should be required to make a therapy clinically viable.

\subsection{Cell growth and proliferation inside scaffolds}

The inhomogeneous scaffolds were used to co-culture with MC3T3-E1 cells to assess if the scaffolds support the growth of cells. The cell attachment, spreading, and growth are important criteria to judge the biocompatibility of a biomaterial. This process is influenced by many properties of biomaterials, including composition, the surface chemical states, and morphology.

It can be observed that cell size was around $5 \sim 30 \mu \mathrm{m}$ and cell number notably increased after extended culture (Figure 4), which confirms that the scaffolds could well support the cell growth. ECM adhesive molecules are concerned with the specific attachment and proliferation of cells on material surface. Collagen and PS are main components of ECM. The interaction of the polar carboxyl, hydroxyl, and amino groups of surface hydrophilic and porous scaffolds with the cells is one possible mechanism to explain the MC3T3-E1 cell affinity. In addition, porosity and pore size in each layer of the scaffolds should be also suited for the cell attachment and migration.

Besides that, it can also be observed from Figure 5 that there were significant differences in the number of proliferated cells between the outer layer and middle layer of scaffolds. As indicated previously, drug-loaded collagen content changed in a increase trend from the outer layer to the middle layer of the scaffolds, and on the other hand, scaffolds had looser pore structures in the middle layer. More cells would grow inwards the layer with both higher porosity and suitable pore size. Therefore, the synergetic effects arisen from composition, structure and certain properties of scaffolds result in significant differences in the number of proliferated cells among layers of scaffolds.

\section{Conclusions}

We developed the inhomogeneous and gradient BG-COL-PS scaffolds embedding the SS using porogen leaching protocol and freeze drying technique. Drug distribution and microstructure could be controlled mainly by regulating the concentrations of drug-loaded collagen microparticles and paraffin spheres as well as freezing temperature for different layers of the scaffolds. It was found that the scaffolds have graded average pore size and porosity, gradient drug distribution, and similar swelling ratio for different layers. In comparision to the scaffolds with homogeneous structure, burst release of drug from inhomogeneous scaffolds was lower and the average release rate was slower after burst release because of gradient drug distribution and graded porous microarchitecture. As well as the achieved scaffolds could well support the cell growth. 


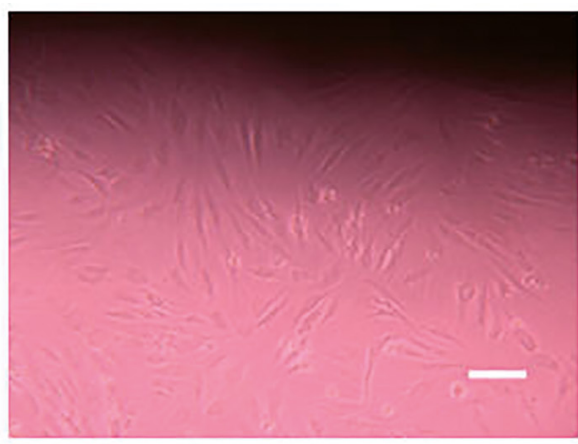

a

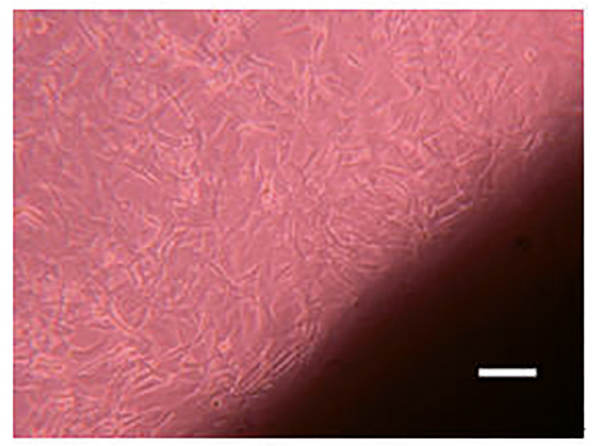

b

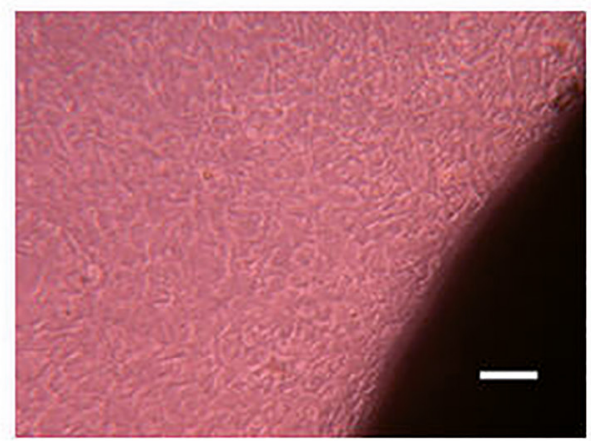

C

Figure 4: Optical micrographs of cells after $1 \mathrm{~d}(\mathrm{a}), 7 \mathrm{~d}$ (b) and $14 \mathrm{~d}$ (c) of culturing with the inhomogeneous scaffolds (scale bar $20 \mu \mathrm{m}$ ). Shadow indicates materials.

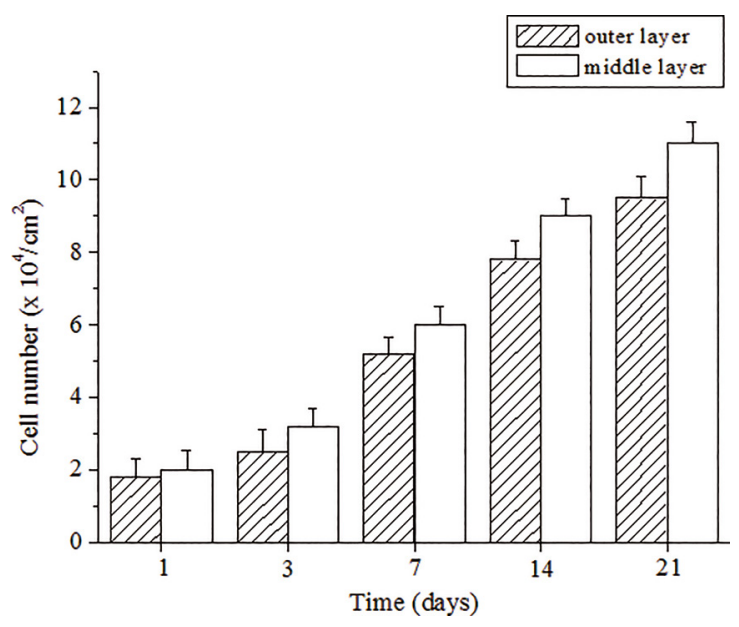

Figure 5: Cell proliferation in different layers of the inhomogeneous scaffolds

\section{Acknowledgments}

This work was financially supported by natural science foundation project of Fujian (Grant no. 2014J05054), scientific research development fundation project of Fujian university of technology (Grant no. GY-Z15093) and scientific research starting foundation project of Fujian university of technology (Grant no. GY-Z0854).

\section{References}

1. Zhu Y, Wan Y, Zhang J, Yin D, Cheng W. Manufacture of layered collagen/chitosan-polycaprolactone scaffolds with biomimetic microarchitecture. Colloids and Surfaces B: Biointerfaces. 2014;113:352-360.

2. Koens MJ, Faraj KA, Wismans RG, van der Vliet JA, Krasznai AG, Cuijpers VM, et al. Controlled fabrication of triple layered and molecularly defined collagen/elastin vascular grafts resembling the native blood vessel. Acta Biomaterialia. 2010;6(12):4666-4674.

3. Knight T, Basu J, Rivera EA, Spencer T, Jain D, Payne R. Fabrication of a multi-layer three-dimensional scaffold with controlled porous micro-architecture for application in small intestine tissue engineering. Cell Adhesion \& Migration. 2013;7(3):267-274.

4. Shalumon KT, Chennazhi KP, Nair SV, Jayakumar R. High thick layer-by-layer 3D multiscale fibrous scaffolds for enhanced cell infiltration and it's potential in tissue engineering. Journal of Biomedical Nanotechnology. 2013;9(12):2117-2122.

5. Sarkar S, Isenberg BC, Hodis E, Leach JB, Desai TA, Wong JY. Fabrication of a layered microstructured polycaprolactone construct for 3-D tissue engineering. Journal of Biomaterials Science. Polymer Edition. 2008;19(10):1347-1362.

6. Lee W, Lee V, Polio S, Keegan P, Lee JH, Fischer K, et al. Ondemand three-dimensional freeform fabrication of multi-layered hydrogel scaffold with fluidic channels. Biotechnology and Bioengineering. 2010;105(6):1178-1186. 
7. Liang C, Li H, Li C, Yang Z, Zhou X, Tao Y, et al. Fabrication of a layered microstructured polymeric microspheres as a cell carrier for nucleus pulposus regeneration. Journal of Biomaterials Science. Polymer Edition. 2012;23(18):2287-2302.

8. Neal RA, Jean A, Park H, Wu PB, Hsiao J, Engelmayr GC Jr, et al. Three-dimensional elastomeric scaffolds designed with cardiac-mimetic structural and mechanical features. Tissue Engineering. Part A. 2013;19(5-6):793-807.
9. Yang CR, Wang YJ, Chen XF. Mineralization regulation and biological influence of bioactive glass-collagen-phosphatidylserine composite scaffolds. Science China. Life Sciences. 2012;55(3):236-240.

10. Wang YZ, Wang JJ, Liang JC, Chen Y. Effects of diosgenin on cell proliferation, differentiation and OPG/RANKL mRNA expression of rat osteoblasts cultured in vitro. China Journal of Traditional Chinese Medicine and Pharmacy. 2010;25(1):134-136.

11. Yang C, Wang J. Preparation and characterization of collagen microspheres for sustained release of steroidal saponins. Materials Research. 2014;17(6):1644-1650. 